\title{
LOCAL UNCERTAINTY INEQUALITIES FOR FOURIER SERIES
}

\author{
JOHN F. PRICE AND PAUL C. RACKI
}

ABSTRACT. Necessary and sufficient conditions are given on $\alpha, \beta$ and $t$ for there to exist a constant $K$ such that

$$
\left(\sum_{n \in E}|\hat{f}(n)|^{2}\right)^{1 / 2} \leqslant K|E|^{\alpha}\left\|f|x|^{\beta}\right\|_{t}
$$

for all $f \in L^{1}\left(T^{d}\right)$ and finite $E \subset Z^{d}$.

1. Introduction. The classical uncertainty principle inequality [2] states that

$$
\||x| f\|_{2}\||y| F\|_{2} \geqslant(d / 4 \pi)\|f\|_{2}^{2}
$$

for all functions $f \in L^{2}\left(\mathbf{R}^{d}\right)$, where the Fourier transform $F$ is defined by

$$
F(y)=\int_{\mathbf{R}^{d}} f(x) \exp (-2 \pi i x \cdot y) d x \quad \text { for } y=\left(y_{1}, \ldots, y_{d}\right) \in \mathbf{R}^{d} .
$$

The natural generalization of this to a product $\mathbf{T}^{d}=(\mathbf{R} / 2 \pi \mathbf{Z})^{d}$ of circle groups fails since $\sum_{n \in \mathbf{Z}^{d}}|n|^{2}|\hat{f}(n)|^{2}=0$ for all constant functions $f \in L^{2}\left(\mathbf{T}^{d}\right)$. (Here and below,

$$
\left.\hat{f}(n)=(2 \pi)^{-d} \int_{\mathbf{T}^{d}} f(x) \exp (-i n . x) d x \quad \text { for } n=\left(n_{1}, \ldots, n_{d}\right) \in \mathbf{Z}^{d} .\right)
$$

Recently, local versions of (1.1) have been developed and applied in quantum physics [3, 4]. Roughly speaking, they assert that if a function is condensed, then not only is its Fourier transform broad, but it cannot be "too" localized. Some of these inequalities involve general $L^{p}$-norms and powers of $|x|$. For example, in [4] it is shown that, given $t \in[1, \infty]$ and $\beta \in \mathbf{R}$, there exists a constant $K$ such that

$$
\left(\int_{E}|F(y)|^{2} d y\right)^{1 / 2} \leqslant K m(E)^{\beta-d / t^{\sharp}}\left\|f|x|^{\beta}\right\|_{t}
$$

for all $f \in L^{2}\left(\mathbf{R}^{d}\right)$ and measurable $E \subset \mathbf{R}^{d}$, with $m(E)<\infty$ if and only if $d / t^{\#}<\beta$ $<d / t^{\prime}$ or $(t, \beta)=(1,0)$ or $(2,0)$, where $t^{\prime}=t /(t-1)$ and $t^{\#}=2 t /(t-2)$. Furthermore, no other power of $m(E)$ apart from $\beta-d / t^{\#}$ is possible. (Global extensions of (1.1) are given in [1].)

Here we establish the following analogous result for $\mathbf{T}^{d}$.

Received by the editors December 12, 1983 and, in revised form, February 27, 1984.

1980 Mathematics Subject Classification. Primary 42B05; Secondary 26D05, 42A05. 
TheOREM. Given $t \in[1, \infty]$ and $\alpha, \beta \in \mathbf{R}$, there exists constant $K$ such that for all functions $f \in L^{1}\left(\mathbf{T}^{d}\right)$,

$$
\left(\sum_{n \in E}|\hat{f}(n)|^{2}\right)^{1 / 2} \leqslant K|E|^{\alpha}\left\|f|x|^{\beta}\right\|_{t}
$$

for all finite $E \subset \mathbf{Z}^{d}$ if and only if $\alpha, \beta, t$ satisfy the following conditions (see Figure 1):

(i) $\beta<d / t^{\prime}$ if $1<t \leqslant \infty$, otherwise $\beta \leqslant d / t^{\prime}$ if $t=1$;

(ii) $\alpha \geqslant \max \left\{0,-1 / t^{\sharp}\right\}$ if $\beta \leqslant 0$ and $1 \leqslant t \leqslant 2$, or $\beta<d / t^{\#}$ and $t<2$;

(iii) $d \alpha \geqslant \beta-d / t^{\sharp}$ if $\max \left\{0, d / t^{\sharp}\right\}<\beta<d / t^{\prime}$.

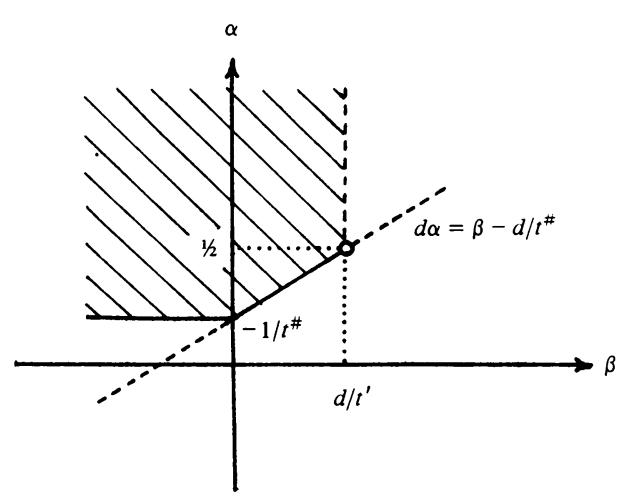

Figure 1a

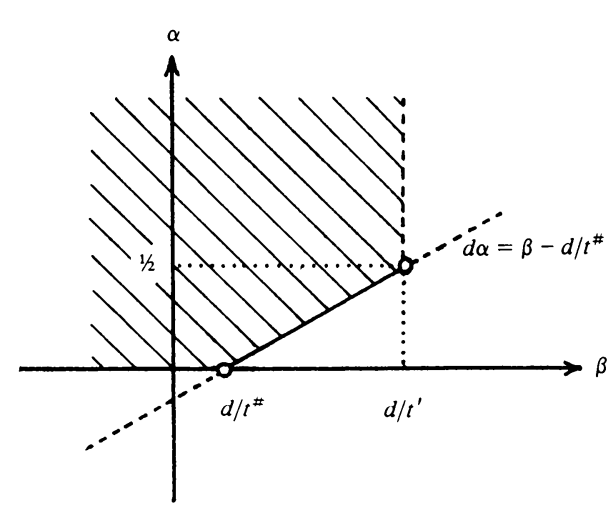

FIGURE 1b

The shaded area of Figure 1(a) is the region of validity of the inequality for $1 \leqslant t \leqslant 2$ with the boundary $\beta=d / t^{\prime}\left(\alpha \geqslant \frac{1}{2}\right)$ being included for $t=1$. When $2<t \leqslant \infty$ the region of validity is the shaded area of Figure 1(b).

2. Proof of sufficiency. Assuming the conditions of the theorem hold, let $N \subset \mathbf{T}^{d}$, $N^{\prime}=\mathbf{T}^{d}-N$ be its complement, and $f$ be a function in $L^{2}\left(\mathbf{T}^{d}\right)$. We thus have $f=f \chi_{N}+f \chi_{N^{\prime}}$, where $\chi_{N}$ is the characteristic function on $N$, so that $\hat{f}=$ $\left(f \chi_{N}\right)^{\hat{n}}+\left(f \chi_{N^{\prime}}\right)^{\hat{*}}$. Hence,

$$
\left(\sum_{n \in E}|\hat{f}(n)|^{2}\right)^{1 / 2} \leqslant\left(\sum_{n \in E} \mid\left(f \chi_{n}\right)^{\left.\hat{(}(n)\right|^{2}}\right)^{1 / 2}+\left(\sum_{n \in E} \mid\left(f \chi_{N^{\prime}}\right)^{\left.\hat{(}(n)\right|^{2}}\right)^{1 / 2}
$$

by Minkowski's inequality; we shall estimate the two quantities separately. First, using Hölder's inequality,

$$
\begin{aligned}
& \left(\sum_{n \in E}\left|\left(f \chi_{N}\right)^{\hat{(}(n)}\right|^{2}\right)^{1 / 2} \leqslant \max \left\{\left|\left(f \chi_{N}\right)^{\hat{n}}(n)\right|: n \in E\right\}|E|^{1 / 2} \leqslant\left\|f \chi_{N}\right\|_{1}|E|^{1 / 2} \\
& =\left\|f \chi_{N}|x|^{\beta}|x|^{-\beta}\right\|_{1}|E|^{1 / 2} \leqslant\left\|f \chi_{N}|x|^{\beta}\right\|_{t}\left\|\chi_{N}|x|^{-\beta}\right\|_{t^{\prime}}|E|^{1 / 2} .
\end{aligned}
$$


Considering the second term of (2.1), we have

$$
\begin{aligned}
\left(\sum_{n \in E}\left|\left(f \chi_{N}^{\prime}\right)^{\wedge}(n)\right|^{2}\right)^{1 / 2} & \leqslant\left(\sum_{n \in Z^{d}}\left|\left(f \chi_{N^{\prime}}\right)^{\wedge}(n)\right|^{2}\right)^{1 / 2}=\left\|f \chi_{N^{\prime}}\right\|_{2} \\
& =\left\|f \chi_{N^{\prime}}|x|^{\beta}|x|^{-\beta}\right\|_{2} \leqslant\left\|f \chi_{N^{\prime}}|x|^{\beta}\right\|_{2 r}\left\|\chi_{N^{\prime}}|x|^{-\beta}\right\|_{2 r^{\prime}} \\
& =\left\|f \chi_{N^{\prime}}|x|^{\beta}\right\|_{t}\left\|\chi_{N^{\prime}}|x|^{-\beta}\right\|_{t^{\sharp}},
\end{aligned}
$$

where we used Hölder's inequality with $r=t / 2$ and assumed $t>2$.

If we assume that $t \in[1,2]$ we estimate the second term as follows:

$$
\begin{aligned}
& \left(\sum_{n \in E}\left|\left(f \chi_{N^{\prime}}\right)^{\hat{x}}(n)\right|^{2}\right)^{1 / 2}=\left(\sum_{n \in E} \mid\left(f \chi_{N^{\prime}}\right)^{\left.\hat{(}(n) \cdot 1\right|^{2}}\right)^{1 / 2} \\
& \leqslant\left(\sum_{n \in E}\left|\left(f \chi_{N^{\prime}}\right)^{\wedge}(n)\right|^{2 r}\right)^{1 / 2 r}\left(\sum_{n \in E} 1^{2 r^{\prime}}\right)^{1 / 2 r^{\prime}} \\
& =\left(\sum_{n \in E}\left|\left(f \chi_{N^{\prime}}\right)^{\hat{x}}(n)\right|^{t^{\prime}}\right)^{1 / t^{\prime}}|E|^{-1 / t^{\#}} \\
& \leqslant\left(\sum_{n \in Z^{d}}\left|\left(f \chi_{N^{\prime}}\right) \hat{(}(n)\right|^{t^{\prime}}\right)^{1 / t^{\prime}}|E|^{-1 / t^{\#}} \\
& \leqslant\left\|f \chi_{N^{\prime}}\right\|_{t}|E|^{-1 / t^{\#}}=\left\|f \chi_{N^{\prime}}|x|^{\beta}|x|^{-\beta}\right\|_{t}|E|^{-1 / t^{\#}} \\
& \leqslant\left\|f \chi_{N^{\prime}}|x|^{\beta}\right\|_{t}\left\|\chi_{N^{\prime}}|x|^{-\beta}\right\|_{\infty}|E|^{-1 / t^{\sharp}},
\end{aligned}
$$

where we used Hölder's inequality with $r=t^{\prime} / 2$, followed by Hausdorff-Young and a final application of Hölder's inequality.

Substituting (2.2)-(2.4) into (2.1), we obtain

$$
\left(\sum_{n \in E}|\hat{f}(n)|^{2}\right)^{1 / 2} \leqslant K\left\|f|x|^{\beta}\right\|_{t}
$$

where $K=K_{1}+K_{2}$, with

$$
\begin{aligned}
& K_{1}=\left\|\chi_{N}|x|^{-\beta}\right\|_{t^{\prime}}|E|^{1 / 2}, \\
& K_{2}= \begin{cases}\left\|\chi_{N^{\prime}}|x|^{-\beta}\right\|_{\infty}|E|^{-1 / t^{\sharp}} & \text { for } 1 \leqslant t \leqslant 2, \\
\left\|\chi_{N^{\prime}}|x|^{-\beta}\right\|_{t^{\sharp}} & \text { for } 2<t \leqslant \infty .\end{cases}
\end{aligned}
$$

Letting $N=\left\{x \in \mathbf{T}^{d}:|x|<a\right\}$ for some $a$, we now evaluate the above norms. First,

$$
\begin{aligned}
\left\|\chi_{N}|x|^{-\beta}\right\|_{t^{\prime}}^{t^{\prime}} & =\int_{|x| \leqslant a}|x|^{-\beta t^{\prime}} d x=W_{d} \int_{0}^{a} r^{-\beta t^{\prime}} r^{d-1} d r \\
& =\left(W_{d} /\left(d-\beta t^{\prime}\right)\right) a^{d-\beta t^{\prime}}
\end{aligned}
$$


as $\beta<d / t^{\prime}$, where $W_{d}=2 \pi^{d / 2} / \Gamma(d / 2)$. Second

$$
\begin{aligned}
\left\|\chi_{N^{\prime}}|x|^{-\beta}\right\|_{t^{\sharp}}^{t^{\sharp}} & =\int_{a \leqslant|x| \leqslant \pi}|x|^{-\beta t^{\sharp}} d x=W_{d} \int_{a}^{\pi} r^{-\beta t^{\sharp}+d-1} d r \\
& =W_{d}\left(\beta t^{\sharp}-d\right)^{-1}\left(a^{d-\beta t^{\sharp}}-\pi^{d-\beta t^{\sharp}}\right) \leqslant W_{d}\left(\beta t^{\sharp}-d\right)^{-1} a^{d-\beta t^{\sharp}},
\end{aligned}
$$

as $\beta>\max \left\{0, d / t^{\sharp}\right\}$. Thirdly if $\beta>0$,

$$
\left\|\chi_{N^{\prime}}|x|^{-\beta}\right\|_{\infty}=\sup \left\{|x|^{-\beta}: a \leqslant|x| \leqslant \pi\right\}=a^{-\beta} .
$$

Now, letting $a=|E|^{-1 / d}$ and substituting (2.6)-(2.8) into (2.5), we obtain

$$
\left(\sum_{n \in E}|\hat{f}(n)|^{2}\right)^{1 / 2} \leqslant K|E|^{\beta / d-1 / t^{*}}\left\|f|x|^{\beta}\right\|_{t},
$$

with $K=K_{1}+K_{2}$, where $K_{1}=\left(W_{d} /\left(d-\beta t^{\prime}\right)\right)^{1 / t^{\prime}}$ and

$$
K_{2}= \begin{cases}1 & \text { for } 1 \leqslant t \leqslant 2, \\ \left(W_{d} /\left(\beta t^{\sharp}-d\right)\right)^{1 / t^{\sharp}} & \text { for } 2<t \leqslant \infty,\end{cases}
$$

provided $\max \left\{0, d / t^{\sharp}\right\}<\beta<d / t^{\prime}$.

Finally, if $\beta \leqslant 0,\left\|\chi_{N^{\prime}}|x|^{-\beta}\right\|_{\infty}=\pi$; hence, $K_{2}=\pi|E|^{-1 / t^{\sharp}}$ and

$$
K_{1}=\left(W_{d} /\left(d-\beta t^{\prime}\right)\right)^{1 / t^{\prime}} a^{d / t^{\prime}-\beta}|E|^{1 / 2}=\left(W_{d} /\left(d-\beta t^{\prime}\right)\right)^{1 / t^{\prime}}|E|^{-1 / t^{\sharp}}
$$

upon letting $a=|E|^{-1 /\left(d-\beta t^{\prime}\right)}$ for $t \neq 1$. If $t=1$,

$$
K_{1}=\left\|\chi_{N}|x|^{-\beta}\right\|_{\infty}|E|^{1 / 2}=a^{-\beta}|E|^{1 / 2}=|E|^{-1 / t^{\#}}
$$

upon letting $a=1$. Thus

$$
\left(\sum_{n \in E}|\hat{f}(n)|^{2}\right)^{1 / 2} \leqslant K|E|^{-1 / t^{*}}\left\|f|x|^{\beta}\right\|_{t}
$$

for $\beta \leqslant 0$ and $1 \leqslant t \leqslant 2$ with $K=K_{1}+K_{2}$, where $K_{2}=\pi$ and

$$
K_{1}= \begin{cases}1 & \text { for } t=1, \\ \left(W_{d} /\left(d-\beta t^{\prime}\right)\right)^{1 / t^{\prime}} & \text { for } 1<t \leqslant 2 .\end{cases}
$$

We thus have the inequality holding along the line segment in the $(\alpha, \beta)$-plane given by $d \alpha=\beta-d / t^{\sharp}$ for $\max \left\{0, d / t^{\sharp}\right\}<\beta<d / t^{\prime}$. When $t=1$ we also have it holding at the endpoint $\beta=d / t^{\prime}=0$ since

$$
\left(\sum_{n \in E}|\hat{f}(n)|^{2}\right)^{1 / 2} \leqslant|E|^{1 / 2}\|\hat{f}\|_{\infty} \leqslant|E|^{1 / 2}\|f\|_{1}
$$

These results may be extended as follows: If

$$
\left(\sum_{n \in E}|\hat{f}(n)|^{2}\right)^{1 / 2} \leqslant K|E|^{\alpha}\left\|f|x|^{\beta}\right\|_{t}
$$

holds for given $(\alpha, \beta, t)$, then it holds for $\left(\alpha^{\prime}, \beta^{\prime}, t^{\prime}\right)$ with $K$ replaced by $K\left\||x|^{\beta-\beta^{\prime}}\right\|_{\infty}$, where $\alpha^{\prime} \geqslant \alpha, \beta^{\prime} \leqslant \beta$ and $t^{\prime} \geqslant t$. To see this, notice that $|E|^{\alpha^{\prime}} \geqslant|E|^{\alpha}$ and $\left\|f|x|^{\beta}\right\|_{t} \leqslant$ $\left\||x|^{\beta-\beta^{\prime}}\right\|_{\infty}\left\|f|x|^{\beta^{\prime}}\right\|_{t^{\prime}}$. 
This leaves only the case $\alpha=0$ and $t \geqslant 2$; it can be established as follows:

$$
\|\hat{f}\|_{2}=\|f\|_{2}=\left\|f|x|^{\beta}|x|^{-\beta}\right\|_{2} \leqslant\left\|f|x|^{\beta}\right\|_{t}\left\||x|^{-\beta}\right\|_{t^{\sharp}}
$$

with

$$
\left\||x|^{-\beta}\right\|_{t^{\sharp}}^{t^{\sharp}}=W_{d} \int_{0}^{\pi} r^{-\beta t^{\sharp}+d-1} d r<\infty \quad \text { for } \beta<d / t^{\sharp}(t>2)
$$

and

$$
\left\||x|^{-\beta}\right\|_{t^{\sharp}}=\sup \left\{|x|^{-\beta}: x \in \mathbf{T}^{d}\right\}<\infty \quad \text { for } \beta \leqslant d / t^{\#}=0(t=2) .
$$

We have thus covered the stated region, and the sufficiency of the conditions is established.

REMARK. In the interior of the region an alternate value of the constant is $K=\left\||x|^{-\beta}\right\|_{2 t /(t+2 \alpha t-2)}$. To see this, notice that

$$
\begin{aligned}
\left(\sum_{n \in E}|\hat{f}(n)|^{2}\right)^{1 / 2} & \leqslant|E|^{1 / 2 r^{\prime}}\left(\sum_{n \in Z^{d}}|\hat{f}(n)|^{2 r}\right)^{1 / 2 r} \\
& \leqslant|E|^{1 / 2 r^{\prime}}\left\|f|x|^{\beta}|x|^{-\beta}\right\|_{(2 r)^{\prime}} \\
& \leqslant|E|^{1 / 2 r^{\prime}}\left\||x|^{-\beta}\right\|_{(2 r)^{\prime p}}\left\|f|x|^{\beta}\right\|_{(2 r)^{\prime p},} \\
& =|E|^{\alpha}\left\||x|^{-\beta}\right\|_{2 t /(t+2 \alpha t-2)}\left\|f|x|^{\beta}\right\|_{t} .
\end{aligned}
$$

3. Proof of necessity. The necessity of the conditions is obtained by ruling out the remaining regions beginning with $\alpha \leqslant 0$. Firstly, we can not have $\alpha<0$, since then $|E|^{\alpha} \rightarrow 0$ as $|E| \rightarrow \infty$. Secondly, considering the case $\alpha=0, \beta=d / t^{\#}$ with $t>2$, the function $f \in L^{1}\left(T^{d}\right)$ defined by

$$
f(x)= \begin{cases}|x|^{-d / 2}|\log | x||^{-1 / 2} & \text { for }|x| \leqslant 1 / 2, \\ 0 & \text { otherwise }\end{cases}
$$

provides a counterexample, since

$$
\|f\|_{2}^{2}=W_{d} \int_{0}^{1 / 2} r^{-1} \log ^{-1} r d r=\infty
$$

while

$$
\left\|f|x|^{d / t^{\sharp}}\right\|_{t}^{t}=W_{d} \int_{0}^{1 / 2} r^{-1}|\log r|^{-t / 2} d r<\infty \quad \text { if } t>2 .
$$

This also rules out the region $\alpha=0, \beta>d / t^{\sharp}$ for $t>2$. Thirdly, consider the region given by $\alpha=0, \beta<d / t^{\#}$ with $1 \leqslant t<2$. Here define $f(x)=|| x|-1|^{-1 / 2}$ for $1 / 2 \leqslant$ $|x| \leqslant 3 / 2$ and 0 otherwise, so that

$$
\|f\|_{2}^{2}=W_{d} \int_{1 / 2}^{3 / 2}|r-1|^{-1} r^{d-1} d r=\infty,
$$

while

$$
\left\|f|x|^{\beta}\right\|_{t}^{t}=W_{d} \int_{1 / 2}^{3 / 2}|r-1|^{-t / 2} r^{\beta t+d-1} d r<\infty \quad \text { for } t<2
$$


Finally, the region given by $\alpha=0, \beta>d / t^{\sharp}=0$ for $t=2$ is ruled out by $f(x)=$ $|x|^{-d / 2}$ since

$$
\|f\|_{2}^{2}=W_{d} \int_{0}^{\pi} r^{-1} d r=\infty
$$

while

$$
\left\|f|x|^{\beta}\right\|_{2}=W_{d} \int_{0}^{\pi} r^{-1+2 \beta} d r<\infty,
$$

which completes the $\alpha \leqslant 0$ case.

Now consider the boundary $\beta \geqslant d / t^{\prime}$; here the function

$$
f_{\varepsilon}(x)= \begin{cases}|x|^{-d}|\log | x||^{-1} & \text { for } 0<\varepsilon \leqslant|x| \leqslant \frac{1}{2}, \\ 0 & \text { otherwise }\end{cases}
$$

provides the required counterexample as $\varepsilon \rightarrow 0$. For assume that $E=\{0\}$; then

$$
\left(\sum_{n \in E}\left|\hat{f}_{\varepsilon}(n)\right|^{2}\right)^{1 / 2}=\left|\hat{f}_{\varepsilon}(0)\right|=\left|W_{d} \int_{\varepsilon}^{1 / 2} r^{-1} \log ^{-1} r d r\right| \rightarrow \infty \quad \text { as } \varepsilon \rightarrow 0,
$$

while

$$
\left\|f_{\varepsilon}|x|^{\beta}\right\|_{t}^{t}=W_{d} \int_{\varepsilon}^{1 / 2} r^{\beta t-d t+d-1}|\log r|^{-t} r d r \leqslant \text { constant }
$$

for all $\varepsilon>0$ if $\beta t-d t+d-1 \leqslant-1$ and $t>1$ or $\beta t-d t+d-1>-1$ and $t=1$. Hence, there is a contradiction for all $\alpha$ if $\beta \geqslant d / t^{\prime}$ and $t>1$ or $\beta>d / t^{\prime}$ and $t=1$.

Consider now the region $d \alpha<\beta-d / t^{\sharp}$ for $\max \left\{0, d / t^{\sharp}\right\} \leqslant \beta<d / t^{\prime}$. Define

$$
f_{N}(x)= \begin{cases}1 & \text { if } x \in \square_{N}, \\ 0 & \text { otherwise }\end{cases}
$$

where

$$
\square_{N}=\left\{x=\left(x_{1}, \ldots, x_{d}\right) \in T^{d}:\left|x_{1}\right| \leqslant 1 / N, \ldots,\left|x_{d}\right| \leqslant 1 / N\right\}
$$

and $N$ is a positive integer. Now

$$
\begin{aligned}
\hat{f}_{N}(n) & =\hat{f}_{N}\left(n_{1}, \ldots, n_{d}\right)=\int_{\square_{N}} e^{-i n_{1} x_{1}} \cdots e^{-i n_{d} x_{d}} d x_{1} \cdots d x_{d} \\
& =2^{d} n_{1}^{-1} \sin n_{1} / N \cdots n_{d}^{-1} \sin n_{d} / N .
\end{aligned}
$$

Thus, letting $E=\left\{n=\left(n_{1}, \ldots, n_{d}\right) \in Z^{d}:\left|n_{1}\right| \leqslant N, \ldots,\left|n_{d}\right| \leqslant N\right\}$, we have

$$
\begin{aligned}
\left(\sum_{n \in E}\left|\hat{f}_{N}(n)\right|^{2}\right)^{1 / 2} & =2^{d}\left(\sum_{\left|n_{1}\right| \leqslant N} \frac{\sin ^{2} n_{1} / N}{n_{1}^{2}} \cdots \sum_{\left|n_{d}\right| \leqslant N} \frac{\sin ^{2} n_{d} / N}{n_{d}^{2}}\right)^{1 / 2} \\
& \sim 1 / N^{d / 2} \text { as } N \rightarrow \infty
\end{aligned}
$$

since

$$
\lim _{N \rightarrow \infty} \sum_{|n| \leqslant N}\left(\frac{n}{N}\right)^{-2} \sin ^{2}\left(\frac{n}{N}\right) N^{-1}=\int_{-1}^{1} x^{-2} \sin ^{2} x d x
$$


while

$$
\begin{aligned}
|E|^{\alpha}\left\|f_{N}|x|^{\beta}\right\|_{t} & =N^{d \alpha}\left(\int_{\square_{N}}|x|^{\beta t} d x\right)^{1 / t} \\
& \leqslant N^{d \alpha}\left(\int_{|x| \leqslant d^{1 / 2} / N}|x|^{\beta t} d x\right)^{1 / t} \\
& =\left(W_{d} /(\beta t+d)\right)^{1 / t} d^{(\beta / 2)+(d / 2 t)} N^{d \alpha-\beta-d / t},
\end{aligned}
$$

assuming $\beta>d / t^{\#}>-d / t$. We thus have a contradiction if $-d / 2>d / \alpha-\beta-d / t$, that is, if $d \alpha<\beta-d / t^{\sharp}$, as required.

Finally, define $g_{N}(x)=f_{N}(x-1), f_{N}$ as above, and $1=(1, \ldots, 1)$, for $N>1$ being an integer. With $E$ as above we have

$$
\left(\sum_{n \in E}\left|\hat{g}_{N}(n)\right|^{2}\right)^{1 / 2} \sim N^{-d / 2} \text { as } N \rightarrow \infty
$$

since $\left|\hat{g}_{N}(n)\right|=\left|\hat{f}_{N}(n)\right|$. Therefore consider, for $\beta<0$,

$$
\begin{aligned}
|E|^{\alpha}\left\|g_{N}|x|^{\beta}\right\|_{t} & =N^{d \alpha}\left(\int_{\square_{N}-1}|x|^{\beta t} d x\right)^{1 / t}=N^{d \alpha}\left(\int_{\square_{N}}|y+1|^{\beta t} d y\right)^{1 / t} \\
& \leqslant\left(\frac{1}{2}\right)^{\beta} N^{d \alpha}\left(\int_{\square_{N}} d y\right)^{1 / t}=2^{d / t-\beta} N^{d \alpha} N^{-d / t}
\end{aligned}
$$

We thus obtain a contradiction as $N \rightarrow \infty$ if $-d / 2>d \alpha-d / t$, that is, $\alpha<-1 / t^{\#}$ for $\beta<0$.

All the required regions are now eliminated and necessity is established which completes the proof of the Theorem.

\section{REFERENCES}

1. M. G. Cowling and J. F. Price, Bandwidth versus time concentration: the Heisenberg-Pauli-Weyl inequality, SIAM J. Math. Anal. 15 (1984), 151-165.

2. D. Dym and H. P. McKean, Fourier series and integrals, Academic Press, New York, 1972.

3. W. G. Faris, Inequalities and uncertainty principles, J. Math. Phys. 19 (1978), 461-466.

4. J. F. Price, Inequalities and local uncertainty principles, J. Math. Phys. 27 (1983), 1711-1714.

School of Mathematics, University of New South Wales, Kensington, New South Wales 2033, Australia 